\title{
Prognosis of patients with acute exacerbation of combined pulmonary fibrosis and emphysema: a retrospective single-centre study
}

Yuichi Ikuyama, Atsuhito Ushiki Đi Makoto Kosaka, Jumpei Akahane, Yuichi Mukai, Taisuke Araki, Yoshiaki Kitaguchi, Kazunari Tateishi, Kazuhisa Urushihata, Masanori Yasuo, Hiroshi Yamamoto and Masayuki Hanaoka

\begin{abstract}
Background: Previous analyses of combined pulmonary fibrosis and emphysema (CPFE) cohorts have provided conflicting data on the survival of patients with CPFE. Therefore, the aim of this study was to investigate the clinical prognosis of acute exacerbations (AE) of CPFE.

Methods: We retrospectively reviewed the medical records of patients who had been treated at the Shinshu University Hospital (Matsumoto, Japan) between 2003 and 2017. We identified 21 patients with AE of CPFE and 41 patients with AE of idiopathic pulmonary fibrosis (IPF) and estimated their prognoses using the Kaplan-Meier method.

Results: Treatment content and respiratory management were not significantly different between the two groups before and after exacerbation. At the time of AE, the median serum Krebs von den Lungen- 6 level was significantly lower in the CPFE group (Krebs von den Lungen-6: $966 \mathrm{U} / \mu \mathrm{L}$; white blood cell count: $8810 / \mu \mathrm{L}$ ) than that in the IPF group (Krebs von den Lungen-6: $2130 \mathrm{U} / \mu \mathrm{L}, p<0.001$; white blood cells: 10809/ $\mu \mathrm{L}, p=0.0096)$. The baseline Gender-Age-Physiology scores were not significantly different between the two groups (CPFE, 4.5 points; IPF, 4.7 points; $p=0.58$ ). Kaplan-Meier curves revealed that the survival time after AE for patients with CPFE was longer than that for patients with IPF $(p<0.001$, logrank test).
\end{abstract}

Conclusions: Survival prognoses after AE were significantly better for patients with CPFE than that for those with IPF. Our findings may improve the medical treatment and respiratory management of patients with AE-CPFE.

Keywords: Acute exacerbation, Combined pulmonary fibrosis and emphysema, Idiopathic pulmonary fibrosis, Prognosis, Emphysema

\section{Background}

Combined pulmonary fibrosis and emphysema (CPFE) is characterised by emphysema in the upper lung and fibrosis in the lower lung on chest high-resolution computed tomography (CT) [1]. Previous analyses of CPFE cohorts have provided conflicting data on the survival

\footnotetext{
* Correspondence: atsuhito@shinshu-u.ac.jp

First Department of Internal Medicine, Shinshu University School of Medicine, 3-1-1, Asahi Matsumoto, Nagano Prefecture 390-8621, Japan
}

implications of emphysema coexisting with fibrosis [2-7]. Similar to patients with idiopathic pulmonary fibrosis (IPF), those with CPFE are also at risk of having episodes of acute exacerbation (AE) of their disease [1]. However, there are limited data available regarding the prognosis of patients with AE-CPFE.

Mejia et al. [7] reported that the overall prognosis in patients who had CPFE with the usual interstitial pneumonia (UIP) pattern was poorer than that of patients

(c) The Author(s). 2020 Open Access This article is licensed under a Creative Commons Attribution 4.0 International License, which permits use, sharing, adaptation, distribution and reproduction in any medium or format, as long as you give appropriate credit to the original author(s) and the source, provide a link to the Creative Commons licence, and indicate if changes were made. The images or other third party material in this article are included in the article's Creative Commons licence, unless indicated otherwise in a credit line to the material. If material is not included in the article's Creative Commons licence and your intended use is not permitted by statutory regulation or exceeds the permitted use, you will need to obtain permission directly from the copyright holder. To view a copy of this licence, visit http://creativecommons.org/licenses/by/4.0/ The Creative Commons Public Domain Dedication waiver (http://creativecommons.org/publicdomain/zero/1.0/) applies to the data made available in this article, unless otherwise stated in a credit line to the data. 
with IPF. However, Kurashima et al. [4] reported that the overall prognosis was better for patients with CPFE than that for those with IPF. They showed that $31.8 \%$ of patients died because of AE-IPF in contrast to $11.1 \%$ who died because of AE-CPFE. The differences in mortality rates between AE-IPF and AE-CPFE may have led to differences in the prognosis between patients with IPF and patients with CPFE. Therefore, we compared differences in the prognosis between patients with AE-CPFE and those with AE-IPF.

\section{Methods}

\section{Study design}

We retrospectively reviewed the medical records of patients with CPFE and IPF, who had been treated at the Shinshu University Hospital (Matsumoto, Japan) between 2003 and 2017. Patients with IPF were included if they met the 2018 clinical practice guideline criteria for IPF, that were established by the American Thoracic Society, European Respiratory Society, Japanese Respiratory Society, and Latin American Thoracic Association [8]. Patients with CPFE were included if they had fibrosis concomitant with UIP, which characterises IPF, because the therapeutic effect in patients with IPF differs from that of other interstitial pneumonias. They were also included if they had a low-attenuation area (LAA) score that was $\geq 7$ points based on chest CT with a slice thickness of $<2.5 \mathrm{~mm}$. The LAA score was calculated as follows: the lungs were divided into six fields (i.e., the upper lobe, middle lobe, and lower lobe of each lung). The upper, middle, and lower lung fields represent the area of the lung above the level of the tracheal carina, the area below the level of the inferior pulmonary vein, and the area between the upper and lower fields, respectively. Emphysema was visually assessed in each patient, and each lung field was assigned a score according to the following criteria established by Goddard et al. [9]: $0=\%$ LAA $<5 \%, 1=\%$ LAA $\geq 5$ and $<25 \%, 2=\%$ LAA $\geq 25$ and $<50 \%, 3=\%$ LAA $\geq 50$ and $<75 \%$, and $4=\%$ LAA $\geq 75 \%$. The scores for each of the six lung fields were summed to obtain the total emphysema score. The CT images were reviewed by two chest radiologists or pulmonologists. All scores were evaluated during the stable period before an AE. Acute exacerbation of IPF was diagnosed based on the diagnostic criteria described by Collard et al. [10] in 2016 as follows: (1) previous or concurrent diagnosis of IPF, (2) acute worsening or development of dyspnoea, typically within a 1-month duration, (3) CT imaging revealed new, bilateral groundglass opacity and/or consolidation superimposed on a background pattern consistent with the UIP pattern, and (4) respiratory deterioration is not fully explained by cardiac failure or fluid overload. Depending on whether an underlying trigger for $\mathrm{AE}$ was determined, the diagnostic criteria categorised $\mathrm{AE}$ as 'triggered $\mathrm{AE}$ ' or 'idiopathic $\mathrm{AE}$ '. In this study, only patients with idiopathic AE were included. An acute exacerbation of CPFE was diagnosed using the same criteria for AE-IPF. Patients who met the criteria for any connective tissue disorder were excluded from the study. At the time of an $\mathrm{AE}$, two chest radiologists or pulmonologists classified the patterns on chest $\mathrm{CT}$ as a peripheral pattern, multifocal pattern, or diffuse pattern, as described in a previous report [11]. The baseline clinical parameters were obtained within 1 month of the initial diagnosis during a stable status. The survival status was obtained from medical records.

\section{Statistical analysis}

Categorical data are presented as the number and percentage, and continuous data are presented as the median and interquartile range.

Differences in the categorical variables between the two groups were compared using the chi-squared test or the $G$ test, and differences in the continuous variables between the two groups were analysed using the MannWhitney $U$ test. The prognosis was estimated using the Kaplan-Meier and log-rank tests. The treatment outcome was assessed as death within 30 days and death within 90 days. A $P$-value of $<0.05$ was considered statistically significant.

\section{Results \\ Baseline clinical characteristics and data of patients with CPFE and IPF}

Based on the selection criteria described previously, the clinical data of 21 patients with AE-CPFE and 41 patients with AE-IPF were enrolled in the present study. One patient with IPF, who had the CT pattern of probable UIP was diagnosed based on surgical lung biopsy findings. The thin-slice CT findings revealed that all other patients with IPF exhibited the UIP pattern. No patient was referred for lung transplantation. Table 1 shows the baseline clinical characteristics and data from patients with IPF and CPFE-UIP before an AE. There were no significant differences in the sex ratio, age, percentage of patients who used corticosteroid or antifibrotic drugs (i.e., pirfenidone or nintedanib), and supplemental oxygen usage between the IPF and CPFE groups. However, cigarette smoking (in pack-years) was significantly higher in the CPFE group than that in the IPF group ( $p=$ 0.0058).

The percentage of predicted forced vital capacity (\%FVC) was significantly higher in the CPFE group than that in the IPF group $(p=0.0069)$. There was no significant difference in the percentage of predicted carbon monoxide diffusing capacity between the two groups. The forced expiratory volume in $1 \mathrm{~s} /$ forced volume vital capacity (i.e., $\mathrm{FEV}_{1} / \mathrm{FVC}$ ) ratio was significantly lower in 
Table 1 Baseline clinical characteristics and data for patients with IPF and patients with CPFE-UIP

\begin{tabular}{|c|c|c|c|}
\hline Parameter & Patients with IPF $(n=41)$ & Patients with CPFE $(n=21)$ & $p$-value \\
\hline \multicolumn{4}{|l|}{ Sex } \\
\hline Male/Female & $34 / 7(83 \% / 17 \%)$ & 19/2 (91\%/9\%) & 0.676 \\
\hline Age (y) & $71(67-75)$ & $74(69-76)$ & 0.14 \\
\hline Cigarette smoking (pack-years) & $32(0.875-44.75)(n=38)$ & $51(29.5-69)(n=19)$ & 0.0058 \\
\hline Patients using corticosteroid drugs & $8(19.5 \%)$ & $6(28.6 \%)$ & 0.419 \\
\hline Patients using immunosuppressant drugs & $1(2 \%)$ & $1(4.8 \%)$ & 0.788 \\
\hline Patients using antifibrotic drugs (e.g. pirfenidone or nintedanib) & $10(24.4 \%)$ & $3(14.3 \%)$ & 0.552 \\
\hline Patients receiving NAC inhalation & $1(0.2 \%)$ & $0(0 \%)$ & 0.73 \\
\hline Patients receiving supplemental oxygen & $12(29.3 \%)$ & $9(42.9 \%)$ & 0.285 \\
\hline LAA score (points) & $1(0-3)$ & $10(9-15)$ & $<0.001$ \\
\hline$\% F V C(\%)$ & $64.5(53.2-80.9)(n=28)$ & $79.3(73.7-90.1)(n=17)$ & 0.0069 \\
\hline$\%$ DLco (\%) & $39.8(29.8-51.9)(n=21)$ & $35.4(30.6-41.1)(n=14)$ & 0.2461 \\
\hline $\mathrm{FEV}_{1} / \mathrm{FVC}$ & 84.75(83-88.4) & $81.2(72.3-87.5)$ & 0.018 \\
\hline GAP score (points) & $4.5(4-6)(n=22)$ & $4.5(4-5)(n=14)$ & 0.58 \\
\hline CPI & $55.4(45.4-61.8)(n=22)$ & $52.6(46.3-58.0)(n=14)$ & 0.41 \\
\hline P/F ratio (Torr) & $331(291-358)(n=26)$ & $328(315-366)(n=17)$ & 0.555 \\
\hline TR-PG (mmHg) & $22.3(15.5-31.2)(n=15)$ & $22.6(18.1-26.1)(n=6)$ & 0.969 \\
\hline WBC count $\left(/ \mathrm{mm}^{3}\right)$ & $7805(6575-8337.5)(n=33)$ & $6780(6270-8000)(n=20)$ & 0.238 \\
\hline CRP (mg/dL) & $0.14(0.06-0.26)(n=29)$ & $0.11(0.04-0.26)(n=19)$ & 0.51 \\
\hline LDH (IU/L) & $222(201-267)(n=29)$ & $234(207-252)(n=19)$ & 0.78 \\
\hline $\mathrm{KL}-6(\mathrm{U} / \mathrm{mL})$ & $1246(899-1494)(n=33)$ & $636.5(442-1102)(n=19)$ & 0.004 \\
\hline $\operatorname{RF}(I U / m L)<10 / \geq 10$ & $25 / 11(n=36)$ a & $9 / 8(n=17)^{\mathrm{b}}$ & 0.242 \\
\hline ANA $<1: 40 / \geq 1: 40$ & $20 / 15(n=35)^{c}$ & $9 / 9(n=18)^{d}$ & 0.621 \\
\hline
\end{tabular}

The parameters are expressed as number (percentage) or median (interquartile range). For insufficient data, the confirmed numbers are indicated by ' $n=$ ' IPF idiopathic pulmonary fibrosis, CPFE combined pulmonary fibrosis and emphysema, UIP usual interstitial pneumonia, NAC n-acetyl-cysteine, $L A A$ low attenuation area, \%FVC percent predicted forced vital capacity, \%DLCo diffusing capacity for carbon monoxide, FEV1/FVC forced expiratory volume in $1 \mathrm{~s} /$ forced volume vital capacity ratio, GAP Gender-Age-Physiology, CPI composite physiologic index, $\mathrm{P} / \mathrm{F}$ ratio of partial pressure arterial oxygen to fraction of inspired oxygen (PaO2/FiO2, $T R-P G$ transtricuspid pressure gradient, $W B C$ white blood cell, $C R P$ C-reactive protein, $L D H$ lactate dehydrogenase, $K L-6$ Krebs von den Lungen-6, $R F$ rheumatoid factor, ANA anti-nuclear antibodies

${ }^{a} 14$ out of 36 patients were data at acute exacerbation. ${ }^{b} 8$ out of 17 patients were data at acute exacerbation. ${ }^{c} 13$ out of 35 patients were data at acute exacerbation. ${ }^{\mathrm{d}} 10$ out of 18 patients were data at acute exacerbation

the CPFE group than that in the IPF group $(p=0.018)$. There were no significant differences in the GenderAge-Physiology (GAP) score, ratio of partial pressure arterial oxygen to fraction of inspired oxygen $(\mathrm{PaO} 2 / \mathrm{FiO} 2$ [i.e., P/F ratio]), composite physiologic index (CPI) score, white blood cell counts, C-reactive protein levels, and lactate dehydrogenase levels between the two groups. Serum levels of Krebs von den Lungen-6 (KL-6) were significantly higher in the IPF group than in the CPFE group $(p=0.004)$.

Echocardiography was performed in 16 patients in the IPF group and 7 in the CPFE group. Among them, the transtricuspid pressure gradient (TR-PG) could be measured in 15 patients and 6 patients, respectively. There was no significant difference in the TR-PG between the two groups.

There were no significant differences between the two groups of patients who were positive for rheumatoid factor and anti-nuclear antibodies. No patients had no physical findings suggestive of collagen disease (e.g. joint pain, rash, or Raynaud's symptoms).

There were no significant differences between the groups in terms of the history of cardiovascular disease ( 4 cases in IPF, 4 cases in CPFE; $p=0.52$ ), malignant tumors ( 5 cases in IPF, 6 cases in CPFE; $p=0.21$ ), diabetes ( 8 cases in IPF, 5 cases in CPFE; $p=0.95$ ), and apoplexia cerebri ( 2 cases in IPF, 1 case in CPFE; $P=0.55$ ).

\section{Laboratory data of patients with CPFE and IPF at the time of the AE}

Table 2 shows the laboratory data of the two groups at the time of the $\mathrm{AE}$. The C-reactive protein level, lactate dehydrogenase level, and $\mathrm{P} / \mathrm{F}$ ratio were not significantly different between the two groups at the time of the AE. However, the serum KL-6 levels and white blood cell counts were significantly lower in the CPFE group than 
Table 2 Laboratory data at the time of acute exacerbation in patients with IPF and CPFE-UIP

\begin{tabular}{|c|c|c|c|}
\hline Parameter & $\begin{array}{l}\text { Patients with IPF } \\
(n=41)\end{array}$ & $\begin{array}{l}\text { Patients with CPFE } \\
(n=21)\end{array}$ & $p$-value \\
\hline$\overline{\text { WBC count }\left(/ \mathrm{mm}^{3}\right)}$ & $10,030(8620-12,500)$ & 88,810 (6460-9320) & 0.0096 \\
\hline CRP (mg/dL) & $5.5(2.4-10.7)$ & $5.85(2.68-7.15)$ & 0.466 \\
\hline LDH (IU/L) & $352(261-453)$ & $289(263-349)$ & 0.151 \\
\hline $\mathrm{KL}-6(\mathrm{U} / \mathrm{mL})$ & $1596(1225-2525)$ & 966 (429-1310) & $<0.001$ \\
\hline P/F ratio (Torr) & $204(148-277)$ & $204(130-261)$ & 0.417 \\
\hline TR-PG (mmHg) & $38.0(33.0-48.1)(n=5)$ & $27.8(25.4-55.0)(n=7)$ & 0.871 \\
\hline $\begin{array}{l}\text { CT pattern: peripheral or } \\
\text { multifocal/diffuse }\end{array}$ & $27 / 14(65.9 \% / 34.1 \%)$ & $17 / 4(89.0 \% / 19.0 \%)$ & 0.345 \\
\hline
\end{tabular}

those in the IPF group at the time of the $\mathrm{AE}(p<0.001$ and $p=0.0096$, respectively). The CT pattern was not significantly different between the two groups at the time of the AE.

Echocardiography was performed in 6 patients in the IPF group and 7 in the CPFE group at the time of acute exacerbation. Among them, TR-PG was measured in 5 and 7 patients, respectively. Although the number of cases was small, there was no significant difference in the TR-PG between the two groups.

\section{Treatment for $A E$ in the two groups}

The treatments for $\mathrm{AE}$ in the two groups are shown in Table 3. All patients in both groups were administered corticosteroids. There were no significant differences in terms of respiratory care, duration of positive-pressure ventilation, or length of stay in the intensive care unit between the two groups. Patients with IPF were more likely to undergo treatment with immunosuppressants than those with CPFE.

\section{Prognosis of the two groups}

The Kaplan-Meier plot in Fig. 1 shows the survival rate of patients with AE-CPFE (solid line) and AE-IPF (dashed line). All patients died of respiratory failure.due to AE. The survival rate was significantly higher in patients with AE-CPFE than in patients with AE-IPF ( $p<$ 0.01 , log-rank test). The 30- and 90-day survival rates for patients in the AE-CPFE group were 95.2\%) and $85.7 \%$, respectively; these values were significantly higher than those (61.0 and $43.9 \%$, respectively) for patients in the AE-IPF group (30 days: $p=0.0066,90$ days: $p=$ 0.0039). Among the patients who died within 90 days, 13 in the AE-IPF group and 2 in the AE-CPFE group had

Table 3 Treatment for acute exacerbation in patients with IPF and CPFE-UIP

\begin{tabular}{|c|c|c|c|}
\hline Treatment & $\begin{array}{l}\text { Patients with IPF } \\
(n=41)\end{array}$ & $\begin{array}{l}\text { Patients with CPFE } \\
(n=21)\end{array}$ & $p$-value \\
\hline Number of patients using corticosteroids & $41(100 \%)$ & $21(100 \%)$ & - \\
\hline Number of patients of using immunosuppressants & $19(46.3 \%)$ & $4(19.0 \%)$ & 0.08 \\
\hline CY & $14(34.1 \%)$ & $3(14.3 \%)$ & \\
\hline CyA & $4(9.7 \%)$ & $2(9.5 \%)$ & \\
\hline TAC & $1(2.4 \%)$ & $0(0 \%)$ & \\
\hline Number of patients receiving respiratory care & & & 0.927 \\
\hline IPPV & $8(19.5 \%)$ & $3(14.3 \%)$ & \\
\hline NPPV & $13(31.7 \%)$ & $7(33.3 \%)$ & \\
\hline HFT & $3(7.3 \%)$ & $1(4.8 \%)$ & \\
\hline Only $\mathrm{O}_{2}$ & $17(41.5 \%)$ & $10(47.6 \%)$ & \\
\hline Duration of positive-pressure ventilation (days) & $9.5(3.75-39.25)(n=20)$ & $5(3.25-7.75)(n=10)$ & 0.628 \\
\hline ICU stay (days) & $10(4-17).(n=13)$ & $4.5(3.25-8)(n=6)$ & 0.122 \\
\hline
\end{tabular}

Note: Most invasive respiratory management methods are listed

The data are expressed as the number (percentage) or as the median (interquartile range)

CPFE combined pulmonary fibrosis and emphysema, $C Y$ cyclophosphamide, CyA cyclosporine, HFT high-flow therapy, ICU intensive care unit, IPF idiopathic

pulmonary fibrosis, IPPV invasive positive-pressure ventilation, NPPV noninvasive positive-pressure ventilation, $\mathrm{O}_{2}$ oxygen, $T A C$ tacrolimus 


\section{Kaplan-Meier survival curve}

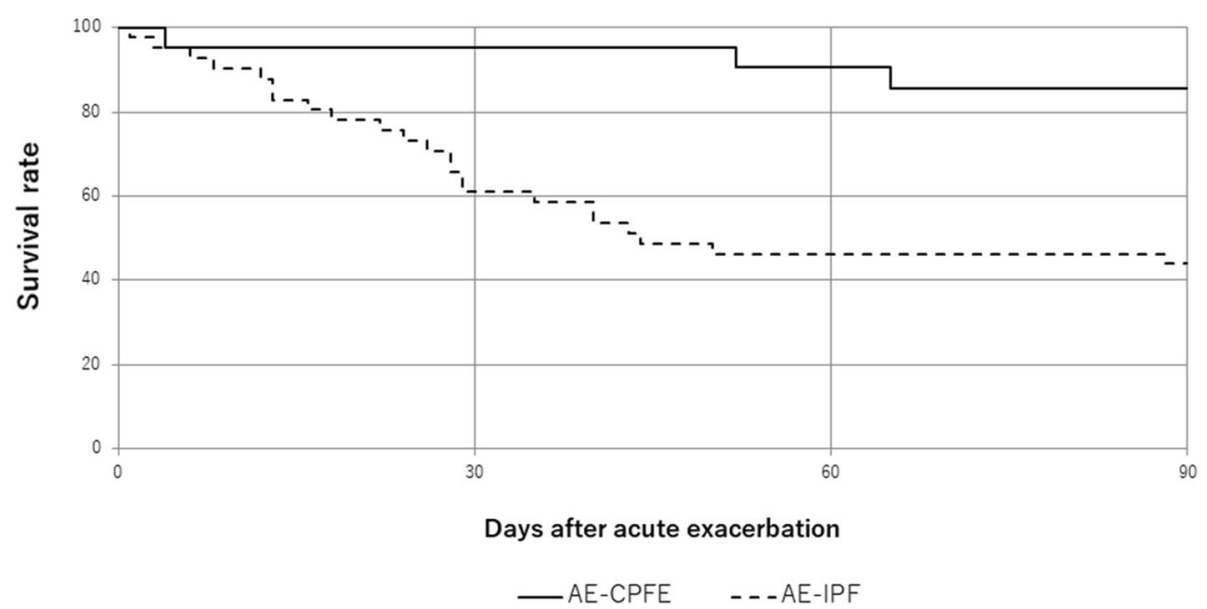

Fig. 1 Survival of patients with AE-CPFE and AE-IPF based on the Kaplan-Meier method. The survival rate of patients with AE-CPFE ( $n=21$; solid line) is significantly lower than that of patients with AE-IPF ( $n=41$; dashed line) $(P<0.001$, log-rank test). The 30- and 90-day survival rates of patients in the AE-CPFE group are 95.2 and $85.7 \%$, respectively. The 30 - and 90-day survival rates of patients in the AE-IPF group were 61.0 and 43.9\%, respectively (30 days: $p=0.0066 ; 90$ days: $p=0.0039$ ). Abbreviations: AE-CPFE, acute exacerbation of combined pulmonary fibrosis and emphysema; AE-IPF, acute exacerbation of idiopathic pulmonary fibrosis

the peripheral pattern, and 10 patients in the AE-IPF group and 1 in the AE-CPFE group had the multifocal/ diffuse pattern $(p=0.345)$.

\section{Discussion}

In this retrospective study, we compared the clinical prognosis of patients with AE-CPFE with that of patients with AE-IPF. Our results demonstrated that the prognosis of AE-CPFE was significantly better than that of AE-IPF.

CPFE is characterised by emphysema in the upper lung and pulmonary fibrosis in the lower lung on CT images. Usual interstitial pneumonia and nonspecific interstitial pneumonia are typically observed in patients with CPF. However, we only compared cases of IPF with cases of CPFE with findings of fibrosis concomitant with UIP on thin-slice chest CT images.

In the present study, patients with AE-CPFE had a significantly better prognosis and a higher survival rate than those with AE-IPF. A recent study demonstrated that the survival rate of AE-IPF was $66 \%$ within 1 month and $41 \%$ within 3 months [12]. This finding was consistent with the findings of our study, which showed a survival rate of $61.0 \%$ within 30 days and $43.9 \%$ within 90 days in patients with AE-IPF.

Previous studies have reported that a low \%FVC and percentage of predicted carbon monoxide diffusing capacity in lung function tests and high KL-6 and lactate dehydrogenase serum levels before $\mathrm{AE}$ are risk factors, that contribute to a poor prognosis in patients with $\mathrm{AE}$ IPF $[13,14]$. The present results also demonstrated that the \%FVC was significantly lower and the levels of KL-6 were significantly higher in the IPF group than in the CPFE group before an AE.

There were no significant differences in the $\mathrm{P} / \mathrm{F}$ ratio, GAP score, CPI and use of supplemental oxygen between the two groups before AE. This finding suggested that the respiratory status of the patients in the two groups was similar before AE. Thus, the disease severity before AE did not differ between the two groups.

Patients with IPF were more likely to undergo treatment with immunosuppressants than those with CPFE. After acute exacerbations, immunosuppressants were added to steroids in cases of poor response. This demonstrated that the clinical course of AE-IPF was worse than that of AE-CPFE.

Yoon et al. [15] reported that IPF developed concomitantly with emphysema in some lung fields in $42 \%$ of their patients, and this finding was based on highresolution $\mathrm{CT}$. In the present study, patients with $\mathrm{CPFE}$ had an LAA score $\geq 7$, which implied that the lung field with emphysema was larger and that with fibrosis was smaller in the CPFE group, than the lung field with emphysema and the lung field with fibrosis in the IPF group.

The KL-6 level at the time of AE was significantly higher in patients with IPF than that in those with CPFE. This finding suggests that the lung injury in patients with IPF was more severe than that in those with CPFE. The area of diffuse alveolar damage, which is a histopathological hallmark of AE-IPF, may be smaller in AE-CPFE than in AEIPF, because of emphysema. We propose that the difference in the composition of fibrosis in the lung fields between the two groups may have led to the difference in prognosis between patients with AE-IPF and patients with AE-CPFE. 
One report [11] indicated that the mortality rate of patients with AE-IPF was higher for individuals with the diffuse ground-glass pattern than for those with the multifocal pattern or peripheral pattern on CT images. The present study revealed that there were no significant differences in the distribution of the CT pattern classifications between the two groups. Moreover, the prognosis of the CPFE group was good, even in patients with the diffuse pattern type. This finding suggests that the pathogenic mechanism of AE-CPFE may differ from that of AE-IPF.

Another possibility is that the pathogenesis of emphysema may antagonise the pathogenesis of ongoing diffuse alveolar damage. Wang et al. [16] demonstrated that urokinase plasminogen activator (uPA) and the urokinase plasminogen activator receptor ( $\mathrm{UPAR}$ ) were overexpressed in alveolar macrophages and in the bronchial epithelium in patients with chronic obstructive pulmonary disease. Moreover, another study [17] demonstrated that lung fibrin clearance was accelerated in a lung-specific inducible uPAexpressing transgenic mouse line. This transgenic mouse line was protected from fibrosis and mortality associated with bleomycin-induced lung injury. Furthermore, the risk of radiation pneumonitis was lower in patients with severe emphysema, such as the patients in our study, than in patients with no underlying lung disease [18]. However, this is a hypothesis, and we intend to test this hypothesis in future studies.

Our study had several limitations. First, it consisted of a single-centre retrospective study, that included a small sample of patients. Some patients were transferred to our hospital after the onset of the AE; thus, their data before the AE were insufficient. Second, only one IPF patient had undergone a surgical lung biopsy, and patients with UIP on high-resolution CT may not necessarily exhibit UIP in pathological examination. However, the patients' clinical characteristics, physiological impairments, and clinical courses were entirely compatible with IPF. Third, only some patients underwent bronchoscopy, and the bronchoalveolar lavage fluid could not be evaluated at the time of the AE. Therefore, the pathogenic mechanisms underlying the differences between AE-CPFE and AE-IPF should be analysed in future research.

\section{Conclusions}

In conclusion, we found that the survival prognosis of AE-CPFE was significantly better than that of AE-IPF for the first time. The differences in the mortality rates between patients with AE-IPF and AE-CPFE may have led to the difference in the prognosis between patients with IPF and those with CPFE. Our findings may improve the medical treatment and respiratory management of patients with AE-CPFE.

\section{Abbreviations}

AE: Acute exacerbation; CPFE: Combined pulmonary fibrosis and emphysema; CT: Computed tomography; FVC: Forced vital capacity; GAP: Gender-Age-Physiology; IPF: Idiopathic pulmonary fibrosis; LAA: Lowattenuation area; LDH: Lactate dehydrogenase; TR-PG: Transtricuspid pressure gradient; UIP: Usual interstitial pneumonia; uPA: Urokinase plasminogen activator; UPAR: Urokinase plasminogen activator receptor

\section{Acknowledgments}

The authors would like to thank Drs N. Kobayashi and Y. Droma (First Department of Internal Medicine at Shinshu University School of Medicine in Matsumoto, Nagano, Japan) for their advice and expertise.

\section{Authors' contributions}

$\mathrm{YI}$ designed the study and wrote the initial draft of the manuscript. AU contributed to the analysis and interpretation of data and assisted in the preparation of the manuscript. MK, JA, YM, TA, YK, KT, KU, MY, HY, and MH contributed to data collection and interpretation and critically reviewed the manuscript. All authors approved the final version of the manuscript and agree to be accountable for all aspects of the work in ensuring that questions related to the accuracy or integrity of any part of the work are appropriately investigated and resolved.

\section{Funding}

This research did not receive any specific grant from funding agencies in the public, commercial, or not-for-profit sectors.

\section{Availability of data and materials \\ Not applicable.}

\section{Ethics approval and consent to participate}

This study was approved by the Ethics Committee of the Shinshu University School of Medicine (Matsumoto, Japan; approval no.: 4063). The authors assert that all procedures contributing to this work complied with the ethical standards of the relevant national and institutional committees on human experimentation and with the Helsinki Declaration of 1975 that was revised in 2008. Informed consent was not required because of the retrospective study design. The study used an opt-out consent model, and patients could choose to opt out at any time and have their data removed from the registry.

\section{Consent for publication}

Not applicable.

\section{Competing interests}

The authors declare that they have no competing interests.

Received: 26 November 2019 Accepted: 13 May 2020

Published online: 20 May 2020

\section{References}

1. Cottin V, Nunes H, Brillet P, Delaval P, Devouassoux G, Tillie-Leblond I, et al. Combined pulmonary fibrosis and emphysema: a distinct underrecognised entity. Eur Respir J. 2005;26:586-93.

2. Ryerson CJ, Hartman T, Elicker BM, Ley B, Lee JS, Abbritti M, et al. Clinical features and outcomes in combined pulmonary fibrosis and emphysema in idiopathic pulmonary fibrosis. Chest. 2013;144:234-40.

3. Jankowich MD, Rounds S. Combined pulmonary fibrosis and emphysema alters physiology but has similar mortality to pulmonary fibrosis without emphysema. Lung. 2010;188:365-73.

4. Kurashima K, Takayanagi N, Tsuchiya N, Kanauchi T, Ueda M, Hoshi T, et al. The effect of emphysema on lung function and survival in patients with idiopathic pulmonary fibrosis. Respirology. 2010;15:843-8.

5. Todd NW, Jeudy J, Lavania S, Franks TJ, Galvin JR, Deepak J, et al. Centrilobular emphysema combined with pulmonary fibrosis results in improved survival. Fibrogenesis Tissue Repair. 2011;4:6.

6. Jacob J, Bartholmai BJ, Rajagopalan S, Kokosi M, Maher TM, Nair A, et al. Functional and prognostic effects when emphysema complicates idiopathic pulmonary fibrosis. Eur Respir J. 2017;50:1700379. 
7. Mejía M, Carrillo G, Rojas-Serrano J, Estrada A, Suarez T, Alonso D, et al. Idiopathic pulmonary fibrosis and emphysema: decreased survival associated with severe pulmonary arterial hypertension. Chest. 2009;136:10-5.

8. Raghu G, Remy-Jardin M, Myers JL, Richeldi L, Ryerson CJ, Lederer DJ, et al. Diagnosis of idiopathic pulmonary fibrosis. An official ATS/ERS/JRS/ALAT clinical practice guideline. Am J Respir Crit Care Med. 2018;198:e44-68.

9. Goddard PR, Nicholson EM, Laszlo G, Watt I. Computed tomography in pulmonary emphysema. Clin Radiol. 1982;33:379-87.

10. Collard HR, Ryerson CJ, Corte TJ, Jenkins G, Kondoh Y, Lederer DJ, et al. Acute exacerbation of idiopathic pulmonary fibrosis. An international working group report. Am J Respir Crit Care Med. 2016;194:265-75.

11. Akira M, Kozuka T, Yamamoto S, Sakatani M. Computed tomography findings in acute exacerbation of idiopathic pulmonary fibrosis. Am J Respir Crit Care Med. 2008;178:372-8.

12. Kondoh Y, Cottin V, Brown KK. Recent lessons learned in the management of acute exacerbation of idiopathic pulmonary fibrosis. Eur Respir Rev. 2017;26:170050.

13. Simon-Blancal V, Freynet $\mathrm{O}$, Nunes $H$, Bouvry D, Naggara $N$, Brillet PY, et al. Acute exacerbation of idiopathic pulmonary fibrosis: outcome and prognostic factors. Respiration. 2012;83:28-35.

14. Kishaba T, Tamaki H, Shimaoka Y, Fukuyama H, Yamashiro S. Staging of acute exacerbation in patients with idiopathic pulmonary fibrosis. Lung. 2014;192:141-9.

15. Yoon HY, Kim TH, Seo JB, Lee SM, Lim S, Lee HN, et al. Effects of emphysema on physiological and prognostic characteristics of lung function in idiopathic pulmonary fibrosis. Respirology. 2019;24:55-62

16. Wang IM, Stepaniants S, Boie Y, Mortimer JR, Kennedy B, Elliott M, et al. Gene expression profiling in patients with chronic obstructive pulmonary disease and lung cancer. Am J Respir Crit Care Med. 2008;177:402-11.

17. Sisson T, Hanson K, Subbotina N, Patwardhan A, Hattori N, Simon RH. Inducible lung-specific urokinase expression reduces fibrosis and mortality after lung injury in mice. Am J Phys Lung Cell Mol Phys. 2002;283:L1023-32.

18. Ishijima M, Nakayama H, Itonaga T, Tajima Y, Shiraishi S, Okubo M, et al. Patients with severe emphysema have a low risk of radiation pneumonitis following stereotactic body radiotherapy. Br J Radiol. 2015;88:20140596.

\section{Publisher's Note}

Springer Nature remains neutral with regard to jurisdictional claims in published maps and institutional affiliations.

Ready to submit your research? Choose BMC and benefit from:

- fast, convenient online submission

- thorough peer review by experienced researchers in your field

- rapid publication on acceptance

- support for research data, including large and complex data types

- gold Open Access which fosters wider collaboration and increased citations

- maximum visibility for your research: over $100 \mathrm{M}$ website views per year

At $\mathrm{BMC}$, research is always in progress.

Learn more biomedcentral.com/submissions 